\title{
Evaluation of the Effect of Different Laser Activated Bleaching Methods on Enamel Susceptibility to Caries; An In Vitro Model
}

\author{
Sajjad Ashnagar ${ }^{1+}$, Abbas Monzavii ${ }^{2+}$, Mehdi Abbasi ${ }^{3}$, Mahdi Aghajani $^{4}{ }^{,}$Nasim Chiniforush ${ }^{2}$ \\ ${ }^{1}$ Department of Periodontics and Oral Medicine, Michigan School of Dentistry, MI, USA \\ ${ }^{2}$ Laser Research Center of Dentistry (LRCD), Dental Research Institute, Tehran University of Medical Sciences, Tehran, Iran \\ ${ }^{3}$ Operative Dentistry Department, School of Dentistry, Tehran University of Medical Sciences, Tehran, Iran \\ ${ }^{4}$ School of Dentistry, Shahid Beheshti University of Medical Sciences, Tehran, Iran
}

\author{
*Correspondence to \\ Nasim Chiniforush, PhD of \\ Laser Dentistry, Laser Research \\ Center of Dentistry (LRCD), \\ Dental Research Institute, Tehran \\ University of Medical Sciences, \\ Tehran, Iran. \\ Postal Code: 1441987566 ; \\ Tel: +98 9124949121; \\ Fax: +98 2122749221; \\ Email: \\ n-chiniforush@farabi.tums.ac.ir \\ ${ }^{+}$Equal contribution.
}

Published online 29 August 2017

\begin{abstract}
Introduction: Today, bleaching is a routine noninvasive alternative for treatment of discolored teeth. The aim of this study was to determine whether conventional or laser activated bleaching predispose teeth to develop caries or not.

Methods: Sixty human molars were mounted on acrylic cylinders and their Knoop microhardness $(\mathrm{KHN})$ as well as DIAGNOdent (DD) values were recorded. They were divided into 4 experimental groups; G1) conventional bleaching with $40 \%$ hydrogen peroxide gel, G2) Diode laser assisted bleaching with same gel, G3) Nd:YAG laser assisted bleaching with the same gel, G4) control group. After bleaching, all samples were subjected to a three day $\mathrm{pH}$ cycling regimen and then, $\mathrm{KHN}$ and DD values were measured.

Results: All groups had significant reduction in $\mathrm{KHN}$ values. It seems that there is no statistically meaningful difference between changes in enamel microhardness of the sample groups and all groups have changed in a similar amount. Reduction of DD scores were significant in Diode laser and conventional groups, however changes in Nd:YAG laser and control groups were not significant. Changes in DD values have followed a similar pattern among groups, except in G1G4 and G2-G4 couples. Conventional and diode laser groups had a meaningful difference in reduction of DD values in comparison with the control group.

Conclusion: It can be concluded that bleaching whether conventional or laser activated, does not make teeth vulnerable to develop carious lesions.

Keywords: Bleaching; Diode laser; Nd:YAG laser, Microhardness; DIAGNOdent
\end{abstract}

\section{Introduction}

In recent years, tooth whitening has become one of the most growing oral care sectors, boosted by patients, demanding both healthy mouth and attractive smile. ${ }^{1}$ As tooth color relies on the composition of tooth tissues, any chemical, mechanical or biological change can damage the esthetic equilibrium of the smile. ${ }^{2}$ Bleaching is a simple, non-restorative and noninvasive treatment for discolored teeth that is capable of satisfaction of the high demanding patients. So it has attracted much interest from the profession.

Bleaching systems today are based on hydrogen peroxide as the active agent and are often activated by heat or light. ${ }^{3}$ Hydrogen peroxide acts as a strong oxidizing agent through the formation of free radicals and reactive molecules which attack dark colored macromolecules and break them into smaller and less colored ones. ${ }^{4}$ Although other products such as carbamide peroxide or sodium perborate are available for bleaching, hydrogen peroxide is still considered to be the most effective and popular agent, since it is available in a range of formulations, concentrations and activation modes. ${ }^{5}$

In-office bleaching can provide significant brightness after only one session of treatment, but achievement of optimum results may require multiple sessions or longer time of application of the bleaching agents. ${ }^{6,7}$ However, this may increase the risk of tooth sensitivity ${ }^{8}$ and cause certain amount of enamel matrix degradation. ${ }^{9}$ Acceleration of hydrogen peroxide decomposition can be carried out through energy absorption from external source of energy like heat or laser, providing better patient compliance and may eradicate the side effects of high concentrated hydrogen peroxide. ${ }^{10,11}$ Because of tooth temperature concerns, lasers are now more preferred. Lasers such as diode (LED), halogen, Nd:YAG etc can be highlighted. 
Bleaching technique of vital teeth requires direct contact of the whitening gel on the outer enamel surface. Oxidizing reaction for an extended period of time can lead to demineralization effects. ${ }^{12,13}$ Demineralization is a process which involves the loss of calcium ions of the surface of calcified dental tissues. Surface morphology of the enamel can be affected, which results in loss of enamel prism peripheries and core. ${ }^{14}$

Some white spots are developed during the whitening process, which disappear after discontinuing the procedure. The concern has been raised whether or not these white spots are precarious lesions. ${ }^{15,16}$ Basting et al suggested that bleaching agents may have a possible influence on active caries lesions in enamel and dentin. ${ }^{17}$ Only few studies have examined the impact of tooth whitening on the susceptibility of enamel to either a carious or erosive challenge. ${ }^{9,18-20}$

Due to controversies in the existing reports, we aimed to evaluate the influence of hydrogen peroxide bleaching with two different light activation methods on enamel susceptibility to caries development, by assessing enamel microhardness and DIAGNOdent (DD) caries values.

\section{Materials and Methods}

Experimental Design

Sixty erupted human third molars, stored in saturated thymol solution at $20^{\circ} \mathrm{C}$ for at least 1 month were used in this study. They were wet-polished with 600 and 1200 grit aluminum oxide abrasive papers. Teeth were mounted onto acrylic cylinders, with an angle ensuring that a flat surface is provided by the buccal aspect of the molar tooth. Microhardness and DD values of the teeth were assessed as the baseline. Samples were then divided into 4 groups $(\mathrm{n}=15)$ : (1) conventional bleaching with $40 \%$ hydrogen peroxide (Opalescence Boost: PF 40, Ultradent, USA), (2) laser assisted bleaching with Diode (CHEESE ${ }^{\text {TM }}$, GIGAA, China), (3) laser assisted bleaching with Nd:YAG (FIDELIS ${ }^{\text {TM }}$, Fotona, Slovenia), and (4) control group. After bleaching was carried out, samples were submitted to artificial early caries solution, during a $\mathrm{pH}$ cycling model. After these treatments, Knoop surface microhardness was determined for each group.

Microhardness and DIAGNOdent Values Measurement Surface microhardness was determined in samples before the bleaching (baseline), and after the $\mathrm{pH}$ cycling model. A Knoop diamond indenter (V-Test, Bareiss Prüfgerätebau $\mathrm{GmbH}$, Germany) was utilized under a load of $50 \mathrm{~g}$ for 8 seconds. Three indentations spaced $100 \mu \mathrm{m}$ from each other were performed. The Knoop hardness indentations were located with their long axis applied perpendicular to the surfaces of the labial enamel. The lengths of the indentations were measured immediately after the indentations had been made.

A DIAGNOdent device (KaVo, Biberach, Germany) was used to assess caries associated with erupted molars. This device uses wavelength of $655 \mathrm{~nm}$ and $5 \mathrm{~mW}$ power. Degrees of caries are reported in values ranged from
0 to 99. Cut-off values for enamel and dentin caries are described by Lussi, which $0-13$ represent healthy tooth substance, 14-20 represent beginning demineralization, 21-29 represent strong demineralization and above 30 represent dentin caries. ${ }^{21}$ Device was calibrated at each session to ceramic standard to ensure reliability of the results. Samples were examined once before the bleaching process and also after the $\mathrm{pH}$ cycling model, by direct and perpendicular contact of the device tip to the study field of the buccal surface.

\section{Bleaching Procedure}

Teeth were bleached according to their bleaching protocol. Facial enamel surfaces were dried with air spray, before the process. Group 1 received a conventional bleaching regimen which included 3 times application of $40 \%$ hydrogen peroxide with gel thickness of 1.5 $\mathrm{mm}$, remaining on the teeth for 20 minutes each time, following manufacturer's instruction. In group 2, teeth were painted with the whitening gel once with gel thickness of $1.5 \mathrm{~mm}$ and irradiated with a diode laser $(810 \mathrm{~nm}$, continuous mode, $1.5 \mathrm{~W})$ with a $400 \mu \mathrm{m}$ fiber tip from a $6 \mathrm{~mm}$ distance. Laser was irradiated for 3 times, 30 seconds each, with 1 minute interval between sessions. Five minutes after last irradiation, whitening agent was washed away with de-ionized and distilled water after 3 minutes of laser application.

In group 3, teeth were painted with the whitening gel once with gel thickness of $1.5 \mathrm{~mm}$ and irradiated with a Nd:YAG laser (1064 nm, pulsed mode, $2.5 \mathrm{~W}$ ) with a $320 \mu \mathrm{m}$ fiber tip from a $6 \mathrm{~mm}$ distance with same protocol as diode laser. Whitening agent was washed away with de-ionized and distilled water after 3 minutes of laser application.

Samples were kept in artificial saliva and stored at $20^{\circ} \mathrm{C}$, to avoid dehydration.

\section{$\mathrm{pH}$-Cycling Process}

A 3-day $\mathrm{pH}$-cycling scheme was performed, with an 18-hour demineralization followed by a 6-hour remineralization, at $37^{\circ} \mathrm{C} .{ }^{22}$ The demineralizing solution at $\mathrm{pH} 4.2$ contained $0.05 \mathrm{M}$ acetic acid, $2.2 \mathrm{mM}$ calcium chloride, $2.2 \mathrm{mM}$ monosodium phosphate, $0.1 \mathrm{M}$ sodium chloride, $2.3 \mathrm{mM}$ sodium fluoride and $0.3 \mathrm{M}$ sodium azide. The remineralizing solution at $\mathrm{pH} 7$ contained the same formulation, except citric acid. A 5-minute wash with the de-ionized and distilled water was done between the demineralization and remineralization cycles and at the end of the protocol. Both remineralizing and demineralizing solutions were changed daily.

\section{Statistical Analysis}

In the descriptive analysis, means and standard errors of microhardness and DIAGNOdent measurements were computed at baseline (before) and directly after the $\mathrm{pH}$ cycling procedure (after). Paired $t$ test was used to compare after and baseline situation of each group. Analysis of variance (ANOVA) followed by Tukey test 
for pair wise comparison was utilized for comparison of each 2 groupg. Commercially available statistical software (SPSSWIN, release 18.0; SPSS, Chicago, IL, USA) was used for all computing.

\section{Results}

Table 1 presents the mean Knoop surface microhardness (KHN) values and standard errors for the experimental groups, before and after the bleaching process. The highest reduction in microhardness of the enamel $(-77.97 \pm 18.76)$ was in conventional group and was followed by diode laser and control groups. The least reduction in enamel microhardness was seen in $\mathrm{Nd}$ :YAG laser group $(-48.19 \pm 14.68)$. All groups had significant reduction in microhardness values.

DD values and standard errors of the study groups can be seen in Table 2. Mean DD value changes are also noted. The highest reduction in DD values was seen in diode laser group (-1.6 \pm 0.66$)$, followed by conventional group and Nd:YAG laser. It was revealed that control group developed some increase in DD values. Reduction of DD scores was significant in diode laser and conventional groups $(P=0.03, P=0.02)$, however changes in $\mathrm{Nd}$ :YAG laser and control groups were not significant $(P=0.17$, $P=0.15)$.

KHN changes and DD changes of the study group during the treatment are compared in Table 3. It seems that there is no statistically meaningful difference between changes in enamel microhardness of sample groups, and all groups have changed in a similar amount. Changes in DD values have followed a similar pattern among groups, except in G1-G4 and G2-G4 couples. Conventional and diode laser groups had a significant difference in reduction of DD values in comparison with control group $(P=0.02$, $P=0.01)$.

\section{Discussion}

Oxidizing reactions during the bleaching process lead to brightening of teeth by decomposition of peroxides into free radicals. Some reports suggest that these oxidation reactions cause enamel structural alterations. ${ }^{23-25}$ These alterations include surface modifications, increases in surface roughness, an increase in the fracture susceptibility, a loss of strength and higher solubility of enamel. ${ }^{15}$ However, all the mentioned topics are on debate. Short time usage of the bleaching agents with lower peroxide concentrations is suggested to be safer compared to the application of high concentration bleaching agents with prolonged contact time. It seems that after a critical concentration, enamel destruction would not be increased anymore and is dependent on application time. ${ }^{26}$

Laser activated bleaching provides improvements in effectiveness, short impact time, and enamel surface protection. ${ }^{10,11}$ The use of high intensity and amplified light has been indicated to accelerate the rate of chemicals involved in bleaching. Zhang et al reported a better bleaching outcome with brighter shades with a safer pulpal temperature rise. ${ }^{27}$ de Magalhães et $\mathrm{al}^{28}$ reported no change in enamel microhardness after bleaching with hydrogen peroxide gel activated with laser.

Table 1. Mean Knoop Surface Microhardness Values $( \pm$ SE) and Mean KHN Changes for Experimental Groups $(n=15)$

\begin{tabular}{lllcc}
\hline Group & $\mathbf{n}$ & Period & Mean KHN \pm SE & Mean KHN Changes \pm SE \\
\hline 1- Conventional & 15 & Before & $262.35 \pm 34.95$ & $-77.97 \pm 18.76$ \\
& & After & $184.38 \pm 21.56$ & $<0.001$ \\
2- Diode laser & 15 & Before & $325.17 \pm 28.48$ & $-73.06 \pm 16.32$ \\
& & After & $252.11 \pm 27.10$ & $-48.19 \pm 14.68$ \\
3- Nd:YAG laser & 15 & Before & $275.63 \pm 24.63$ & 0.005 \\
& & After & $227.44 \pm 22.9$ & $-60.64 \pm 14.79$ \\
\hline
\end{tabular}

Abbreviations: KHN, Knoop surface microhardness.

Table 2. Mean DIAGNOdent Values $(\mathrm{DD} \pm S E$ ) and Mean DIAGNOdent Score Changes for Experimental Groups ( $\mathrm{n}=15$

\begin{tabular}{|c|c|c|c|c|c|}
\hline Group & $\mathbf{n}$ & Period & Mean DD Scores \pm SE & Mean DD Changes \pm SE & $P$ Value \\
\hline \multirow{2}{*}{ 1- Conventional } & \multirow{2}{*}{15} & Before & $7.2 \pm 0.76$ & \multirow{2}{*}{$-1.53 \pm 0.59$} & \multirow{2}{*}{0.02} \\
\hline & & After & $5.66 \pm 0.42$ & & \\
\hline \multirow{2}{*}{ 2- Diode laser } & \multirow{2}{*}{15} & Before & $7.46 \pm 0.96$ & \multirow{2}{*}{$-1.6 \pm 0.66$} & \multirow{2}{*}{0.03} \\
\hline & & After & $5.86 \pm 0.56$ & & \\
\hline \multirow{2}{*}{ 3- Nd:YAG laser } & \multirow{2}{*}{15} & Before & $7.06 \pm 0.62$ & \multirow{2}{*}{$-0.66 \pm 0.46$} & \multirow{2}{*}{0.17} \\
\hline & & After & $6.4 \pm 0.64$ & & \\
\hline \multirow{2}{*}{ 4- Control } & \multirow{2}{*}{15} & Before & $7.13 \pm 0.63$ & \multirow{2}{*}{$+1.13 \pm 0.74$} & \multirow{2}{*}{0.15} \\
\hline & & After & $8.2 \pm 0.67$ & & \\
\hline
\end{tabular}

Abbreviation: DD, DIAGNOdent. 
Table 3. Comparison of KHN and DD Changes Among Experimental Groups, During the Treatment

\begin{tabular}{|c|c|c|c|c|}
\hline Group Comparison & Difference in KHN changes $\pm \mathrm{SE}$ & $P$ Value & Difference in DD changes \pm SE & $P$ Value \\
\hline G1-G2 & $-4.9 \pm 22.94857$ & 0.99 & $0.06 \pm 0.88425$ & 1.00 \\
\hline G1-G3 & $-29.77 \pm 22.94857$ & 0.56 & $-0.86 \pm 0.88425$ & 0.76 \\
\hline G1-G4 & $-17.32 \pm 22.94857$ & 0.87 & $-2.66 \pm 0.88425$ & 0.02 \\
\hline G2-G3 & $-24.86 \pm 22.94857$ & 0.71 & $-0.93 \pm 0.88425$ & 0.71 \\
\hline G2-G4 & $-12.41 \pm 22.94857$ & 0.94 & $-2.73 \pm 0.88425$ & 0.01 \\
\hline G3-G4 & $+12.45 \pm 22.94857$ & 0.94 & $-1.8 \pm 0.88425$ & 0.18 \\
\hline
\end{tabular}

Abbreviations: KHN, Knoop surface microhardness; DD, DIAGNOdent; G1, conventional bleaching; G2, laser assisted laser bleaching (diode); G3, laser assisted bleaching (Nd:YAG); G4, control group.

Yet, it is a question of whether tooth bleaching and subsequent mineral loss of enamel would hasten caries formation process and make the tooth more susceptible to acidic challenges or not. Some studies reported that the bleaching agents develop microstructural and chemical changes in enamel, similar to initial carious lesions. ${ }^{15,16}$ Besides, there is a growing trend in laser activated bleaching among clinicians.

In the present study, the effect of different bleaching activation methods including conventional and two laser activated methods on enamel microhardness, after a cariogenic simulator, was assessed. Baseline microhardness and DD values were measured, and after completing the bleaching process, teeth were submitted to $\mathrm{pH}$ cycling regimen. This cycle, included an 18-hour immersion in a demineralizing solution with $\mathrm{pH}$ of 4.2 , containing calcium, phosphate and fluoride ions. It was followed by a 6-hour remineralizing solution with $\mathrm{pH}$ of 6.75. This regimen almost inducted a model of carious challenge. Neutral remineralizing solution was supposed to mimic saliva, providing mineral content needed for repair of initial demineralized areas. Afterwards, microhardness and DD values were reassessed.

Although the microhardness test does not provide specific information on changes that happen to a material, it is commonly used to measure changes in tooth structures after experiments involving demineralization and remineralization. ${ }^{29}$ Changes in enamel can be efficiently studied by this test.

Our results suggest that the bleaching does not make teeth vulnerable to caries. There was no significant difference between the control group and any of the bleached groups. All groups exhibited a significant change in enamel microhardness after cariogenic challenge and no significant difference was seen in magnitude of these changes. The findings of this study are in accordance with the results of de Menezes et $\mathrm{al}^{20}$ study, in which they assessed the effect of different concentrations of carbamide peroxide gel on enamel susceptibility to caries like lesions. They also used similar $\mathrm{pH}$ cycling regimen to induce caries-like lesions, involving 4 cycles of demineralizing solution with $\mathrm{pH}$ of 5 and remineralizing solution with $\mathrm{pH}$ of 7. de Menezes et al found that bleaching does not seem to pose any extra risk to caries lesion formation in enamel. Furthermore, Burgmaier et $\mathrm{al}^{30}$ assessed the effect of an acidic challenge on $10 \%$ carbamide peroxide bleached enamel samples and found no significant difference in surface microhardness between bleached and control samples. Moreover, Pretty et $\mathrm{al}^{18}$ found that bleaching with 10\%-22\% carbamide peroxide does not make enamel or dentine more susceptible to acid erosion or caries lesion formation, by using quantitative lightinduced fluorescence and transverse microradiography. In addition, Al-Qunaian (19) supported the idea that bleaching does not make tooth more vulnerable to caries formation via in vitro microbial caries model, after bleaching with $10 \%$ and $20 \%$ carbamide peroxide and $35 \%$ hydrogen peroxide. This finding was confirmed in rat molars with $10 \%$ carbamide peroxide, by Kraigher et al. $^{31}$

However, regarding the application time of the bleached groups, a relative but not definite pattern in KHN reduction could be found. Following manufacturers' protocol, hydrogen peroxide gel had a direct contact with enamel surface for 60 minutes. Laser treated group had a contact time of 9 minutes. Regarding KHN values, the highest reduction was seen in conventional therapy $(-77.97 \pm 18.76)$ and laser treated group had less subsidence of KHN during the bleaching process. Nd:YAG laser had the least reduction of microhardness among the experimental groups, even less than the control group which did not received any bleaching treatment. As differences of KHN changes had no statistically significant difference (Table 3), no reliable inferences could be drawn. de Menezes et $\mathrm{a}^{20}$ also found that after caries induction phase, an experimental group bleached with $16 \%$ carbamide peroxide exhibited higher KHN values compared to unbleached groups.

Some limitations could explain the non-statistically significant results among the groups. The number of sample to thoroughly investigated the effect of bleaching methods on caries susceptibility was relatively small $(n=15)$. There was also a large variability among the samples. This variation could be related to different race, age or area of habitance of teeth donors. This diversity in baseline KHN demanded at least 90 samples per group which could not be covered by this study. Higher number of samples with more unified values of initial KHN, might result in a reliable pattern of $\mathrm{KHN}$ changes and therefore, the effects of each intervention could be compared precisely. Also, the demineralizing solution used in this study might have developed an aggressive mineral loss 
and also impaired surface mineral recovery. Thus, the enamel prisms could be disorganized and disrupted, as reported by several authors. ${ }^{32-35}$

DIAGNOdent is a device which measures caries level of teeth by emitting a laser beam and analyzing the reflected light, while directly in contact with the enamel surface. Data acquired by this device are proportional and not pure values. It was assumed that induction of caries would result in a general increase in DD values. Surprisingly, the DD values were increased in all bleached groups of this study. Highest reduction was seen in conventional bleaching group $(P=0.02)$. In contrast, the control group showed increased DD values. Comparing DD changes among the groups, only conventional and diode laser groups had a meaningful difference in reduction of DD compared to the control group. Obviously, higher decrease in caries values is not expected after the oxidizing challenge exerted during the bleaching process. The underlying mechanism of such finding is that DIAGNOdent device detects caries levels by measuring fluorescent molecules in the decayed teeth. Bleaching targets those fluorescent molecules and decreases fluorescence values of the teeth. However decayed structures remain unaffected. This finding is in accordance with previous studies which concluded that fluorescence level of the teeth decrease or remain stable during the bleaching process, and DIAGNOdent as a caries measurement device would bring unreliable values for recent bleached teeth. ${ }^{36-38}$ However, an additional measurement of DD values, after bleaching and prior to $\mathrm{pH}$ cycling could better illustrate the precise effect of bleaching on DD values.

Further studies are needed to clarify the clinical relevance of the findings of this invitro study. Invitro studies cannot be fully representative of the oral cavity as continuous supply of saliva containing various minerals, oral hygiene situation and changes in saliva flow might interfere with this simulation.

\section{Conclusion}

Concerning limitation of this study, it can be concluded that bleaching whether conventional or laser activated method, does not make teeth vulnerable to develop carious lesions.

\section{Ethical Considerations}

Not applicable.

\section{Conflict of Interests}

The authors have no conflict of interest to declare.

\section{References}

1. Anaraki SN, Shahabi S, Chiniforush N, Nokhbatolfoghahaei $\mathrm{H}$, Assadian H, Yousefi B. Evaluation of the effects of conventional versus laser bleaching techniques on enamel microroughness. Lasers Med Sci. 2015;30(3):1013-1018. doi:10.1007/s10103-014-1523-6.

2. Mirzaie M, Yassini E, Ganji S, Moradi Z, Chiniforush N. A comparative study of enamel surface roughness after bleaching with diode laser and Nd: YAG laser. J Lasers Med Sci. 2016;7(3):197-200. doi:10.15171/jlms.2016.34.

3. Kiomarsi N, Salim S, Sarraf P, Javad-Kharazifard M, Chiniforush N. Evaluation of the Diode laser $(810 \mathrm{~nm}$, $980 \mathrm{~nm})$ on dentin tubule diameter following internal bleaching. J Clin Exp Dent. 2016;8(3):e241-e2455. doi: 10.4317/jced.52666.

4. Dahl J, Pallesen U. Tooth bleaching - a critical review of the biological aspects. Crit Rev Oral Biol Med. 2003;14(4):292304.

5. Barghi N. Making a clinical decision for vital tooth bleaching: at-home or in-office? Compend Contin Educ Dent. 1998;19(8):831-838.

6. Torres CRG, Wiegand A, Sener B, Attin T. Influence of chemical activation of a $35 \%$ hydrogen peroxide bleaching gel on its penetration and efficacy-In vitro study. J Dent. 2010;38(10):838-846.

7. Haywood VB. Number of in-office light-activated bleaching treatments needed to achieve patient satisfaction. Quintessence Int. 2006;37:115-120.

8. Marson F, Sensi L, Vieira L, Araújo E. Clinical evaluation of in-office dental bleaching treatments with and without the use of light-activation sources. Oper Dent. 2008;33(1):1522. doi:10.2341/07-57.

9. Tezel H, Atalayin C, Erturk O, Karasulu E. Susceptibility of enamel treated with bleaching agents to mineral loss after cariogenic challenge. Int J Dent. 2011;2011:953835. doi:10.1155/2011/953835.

10. Dostalova T, Jelinkova H, Housova D, et al. Diode laseractivated bleaching. Braz Dent J. 2004;15:SI-3.

11. Goharkhay K, Schoop U, Wernisch J, Hartl S, De Moor R, Moritz A. Frequency doubled neodymium: yttriumaluminum-garnet and diode laser-activated power bleaching- $\mathrm{pH}$, environmental scanning electron microscopy, and colorimetric in vitro evaluations. Lasers Med Sci. 2009;24(3):339-346. doi:10.1007/s10103-0080567-x.

12. Oliveira Rd, Paes Leme AF, Giannini M. Effect of a carbamide peroxide bleaching gel containing calcium or fluoride on human enamel surface microhardness. Braz Dent J. 2005;16(2):103-106.

13. Faraoni-Romano JJ, Turssi CP, Serra MC. Concentrationdependent effect of bleaching agents on microhardness and roughness of enamel and dentin. Am J Dent. 2007;20(1):3134.

14. Flaitz C, Hicks M. Effects of carbamide peroxide whitening agents on enamel surfaces and caries-like lesion formation: an SEM and polarized light microscopic in vitro study. ASDC J Dent Child. 1995;63(4):249-256.

15. Oltu Ü, Gürgan S. Effects of three concentrations of carbamide peroxide on the structure of enamel. J Oral Rehabil. 2000;27(4):332-340.

16. Potočnik I, Kosec L, Gašperšič D. Effect of $10 \%$ carbamide peroxide bleaching gel on enamel microhardness, microstructure, and mineral content. $J$ Endod. 2000;26(4):203-206.

17. Basting R, Rodrigues JA, Serra M. The effect of $10 \%$ carbamide peroxide bleaching material on microhardness of sound and demineralized enamel and dentin in situ. Oper Dent. 2000;26(6):531-539.

18. Pretty I, Edgar W, Higham S. The effect of bleaching on enamel susceptibility to acid erosion and demineralisation. B Dent J. 2005;198(5):285-290. 
19. Al-Qunaian T. The effect of whitening agents on caries susceptibility of human enamel. Oper Dent. 2005;30(2):265270.

20. de Menezes M, Turssi CP, Faraoni-Romano JJ, Serra MC. Susceptibility of bleached enamel and root dentin to artificially formed caries-like lesions. Am J Dent. 2007;20(3):173-176

21. Lussi A. Validity of diagnostic and treatment decisions of fissure caries. Caries Res. 1991;25(4):296-303.

22. Liu J-f, Liu Y, Stephen HC-Y. Optimal Er: YAG laser energy for preventing enamel demineralization. J Dent. 2006;34(1):62-66.

23. Spalding M, Taveira LA, de Assis GF. Scanning electron microscopy study of dental enamel surface exposed to $35 \%$ hydrogen peroxide: alone, with saliva, and with $10 \%$ carbamide peroxide. J Esthet Restor Dent. 2003;15(3):154165. doi:10.1111/j.1708-8240.2003.tb00185.x.

24. Lopes GC, Bonissoni L, Baratieri LN, Vieira LCC, Monteiro S. Effect of bleaching agents on the hardness and morphology of enamel. J Esthet Restor Dent. 2002;14(1):2430. doi:10.1111/j.1708-8240.2002.tb00144.x.

25. Rotstein I, Dankner E, Goldman A, Heling I, Stabholz A, Zalkind M. Histochemical analysis of dental hard tissues following bleaching. J Endod. 1996;22(1):23-26.

26. Bistey T, Nagy IP, Simó A, Hegedűs C. In vitro FT-IR study of the effects of hydrogen peroxide on superficial tooth enamel. J Dent. 2007;35(4):325-330.

27. Zhang C, Wang X, Kinoshita J-I, et al. Effects of KTP laser irradiation, diode laser, and LED on tooth bleaching: a comparative study. Photomed Laser Surg. 2007;25(2):91-95. doi:10.1089/pho.2006.2025.

28. de Magalhães MT, Basting RT, de Almeida ER, Pelino JEP. Diode laser effect on enamel microhardness after dental bleaching associated with fluoride. Photomed Laser Surg. 2009;27(6):937-941. doi:10.1089/pho.2008.2440.
29. Ryge G, Foley DE, Fairhurst CW. Micro-indentation hardness. J Dent Res. 1961;40(6):1116-1126.

30. Burgmaier GM, Schulze I, Attin T. Fluoride uptake and development of artificial erosions in bleached and fluoridated enamel in vitro. J Oral Rehabil. 2002;29(9):799804.

31. Kraigher A, Van der Veen M, Potočnik I. Caries occurrence in rats after bleaching with $10 \%$ carbamide peroxide in vivo. Caries Res. 2005;40(1):77-80.

32. Pinto CF, Oliveira Rd, Cavalli V, Giannini M. Peroxide bleaching agent effects on enamel surface microhardness, roughness and morphology. Braz Oral Res. 2004;18(4):306311.

33. Perdigao J, Francci C, Swift E Jr, Ambrose W, Lopes M. Ultra-morphological study of the interaction of dental adhesives with carbamide peroxide-bleached enamel. Am J Dent. 1998;11(6):291-301.

34. Türkün M, Sevgican F, Pehlivan Y, Aktener BO. Effects of $10 \%$ carbamide peroxide on the enamel surface morphology: a scanning electron microscopy study. J Esthet Restor Dent. 2002;14(4):238-44. doi:10.1111/j.1708-8240.2002. tb00169.x.

35. Pinto CF, Leme AFP, Cavalli V, Giannini M. Effect of $10 \%$ carbamide peroxide bleaching on sound and artificial enamel carious lesions. Braz Dent J. 2009;20(1):48-53.

36. Falconer D, Hamilton J, Gregory W, Stoffers K. Tooth Bleaching and Its Effect on Diagnosing P\&F Caries. ADEA/AADR/CADR Meeting \& Exhibition; Orlando, March 2006.

37. Hitij T, Fidler A. Effect of dental material fluorescence on DIAGNOdent readings. Acta Odontol. 2008;66(1):13-17.

38. Rashidian A, Shahbazi Moghaddam M, Nadarvand S, Afsharian Zadeh M. Evaluation of the effect of aging, staining and bleaching on diagnodent findings in composite fillings (in vitro). Res Dent Sci. 2013;9(4):206-212. 